\title{
Prevalence and Determinants of Cognitive Impairment in Elderly Patients With Heart Failure - Pilot Study in a Geriatric Hospital -
}

\author{
Shutaro Futami, MD; Joji Ishikawa, MD, PhD; Chihiro Jubishi, MD; Ruri Shimizu, MD; \\ Teppei Murata, MD; Rie Aoyama, MD, PhD; Kazumasa Harada, MD, PhD
}

\begin{abstract}
Background: Elderly patients admitted to hospital with heart failure (HF) often have cognitive impairment, but the association between these conditions is unclear.

Methods and Results: We enrolled 43 patients admitted to a geriatric hospital with HF. We evaluated echocardiography, Mini Mental State Examination (MMSE), and extracellular water/total body water (ECW/TBW) ratio (Inbody S10). Mean age was $85.1 \pm 8.0$ years (range, $60-99$ years) and $44.2 \%$ of the patients were men. Mean MMSE score was $20.5 \pm 5.4$, with $66.7 \%$ of the patients showing cognitive impairment (MMSE $\leq 23)$. There was a significant negative correlation of MMSE score with age $(r=-0.344, P=0.032)$, regular alcohol drinking $(r=0.437, P=0.007)$, uric acid level $(r=0.413, P=0.010)$, and ECW/TBW ratio $(r=-0.437, P=0.007)$. On stepwise regression analysis including these covariates, MMSE score was significantly associated with the ECW/TBW ratio $(\beta=0.443$, $P=0.009$ ). When several echocardiography parameters (i.e., end-diastolic left ventricular volume, $r=0.327, P=0.048$; left atrial volume index, $r=-0.411, P=0.012$; and transmitral inflow $A$ wave velocity, $r=-0.625, P=0.001$ ) were added to the model, MMSE score was found to be related to the $A$ wave $(P=0.001)$ and to atrial volume index $(P=0.015)$, which are measures of diastolic function.
\end{abstract}

Conclusions: In elderly patients with HF, cognitive function might be influenced by body water distribution and diastolic heart function.

Key Words: Cognitive function; Congestive heart failure; Dementia; Elderly; Fluid balance

I $\mathrm{t}$ is important to maintain the quality of life for elderly persons while reducing mortality from heart disease. The incidence of heart failure (HF) increases with aging, and cognitive impairment is sometimes observed in elderly patients with HF. An association has been noted between left ventricular (LV) systolic dysfunction and cognitive impairment. ${ }^{\mathbf{1}, \mathbf{2}} \mathrm{We}$ recently reported that cognitive function measured in subjects in the community using the Montreal cognitive assessment, which assesses mild cognitive impairment, was associated with peak $\dot{\mathrm{VO}}_{2} /$ pulse rate ratio, a marker of LV stroke volume. ${ }^{3}$ In addition, Sabayan et al noted an association between LV systolic function and structural and functional features of cerebral aging on high-resolution brain magnetic resonance imaging (MRI). ${ }^{4}$

Cognitive impairment, however, has been observed in $\mathrm{HF}$ patients with either a reduced or preserved $\mathrm{LV}$ ejection fraction (HFrEF or HFpEF, respectively) and some studies have indicated an association between LV diastolic dysfunction and cognitive impairment..$^{\mathbf{5} 6}$ Moreover, the mechanisms linking $\mathrm{HF}$ to cognitive impairment remain unclear.
HF causes systemic congestion, and it is necessary to control the fluid balance in these patients. Systemic congestion caused by HF has been shown to be associated with dysfunction/failure of extracardiac organs such as the kidneys and intestines. ${ }^{7-9}$ However, there is little information on the association between systemic congestion and cognitive impairment. Therefore, the aim of the present study was to evaluate whether cognitive impairment is associated with the extracellular body water (ECW) status in elderly HF patients admitted to a geriatric hospital.

\section{Methods}

\section{Subjects}

We enrolled consecutive patients admitted to a geriatric hospital with decompensated HF $(n=452)$ between July 2015 and July 2016 after exclusion of patients with multiple hospitalizations. We were able to obtain informed consent from 50 patients. The number of patients analyzed in the present study was 43 , after excluding the following patients:

Received October 29, 2019; revised manuscript received February 12, 2020; accepted March 4, 2020; J-STAGE Advance Publication released online March 26, 2020 Time for primary review: 24 days

Tokyo Metropolitan Geriatric Hospital and Institute of Gerontology, Department of Cardiology, Tokyo, Japan

Current address: Department of Hematology, Oncology and Cardiovascular Medicine, Kyushu University Hospital, Fukuoka (S.F.), Japan

Mailing address: Joji Ishikawa, MD, PhD, Tokyo Metropolitan Geriatric Hospital, Department of Cardiology, 35-2 Sakae-cho, Itabashi-ku, Tokyo 173-0015, Japan. E-mail: joji_ishikawa@tmghig.jp

All rights are reserved to the Japanese Circulation Society. For permissions, please e-mail: cr@j-circ.or.jp

ISSN-2434-0790 


\begin{tabular}{lc|}
\hline Table 1. Patient Characteristics & \\
Age (years) & $85.1 \pm 8.9$ \\
Male & 42.0 \\
Height (cm) & $150.5 \pm 8.5$ \\
Body weight (kg) & $49.8 \pm 12.5$ \\
BMI (kg/m²) & $21.8 \pm 3.9$ \\
Alcohol drinking & 20.9 \\
Smoking status & \\
Ex-smoker & 25.6 \\
Current smoker & 11.6 \\
HFpEF & 51.2 \\
Hypertension & 95.3 \\
Dyslipidemia & 32.6 \\
Diabetes & 18.6 \\
Atrial fibrillation & 37.2 \\
History of stroke & 11.6 \\
SBP (mmHg) & $119.2 \pm 17.2$ \\
DBP (mmHg) & $59.8 \pm 14.0$ \\
Pulse rate (beats/min) & $75.0 \pm 16.1$ \\
\hline Antihypertensive drug use & 90.7 \\
ACEI or ARB & 60.5 \\
$\beta$-blocker & 60.5 \\
CCB & 41.9 \\
Antiplatelet agent & 44.2 \\
Warfarin & 14.0 \\
Direct oral anticoagulant & 11.6 \\
MMSE score & $20.5 \pm 5.4$ \\
MMSE score $\leq 23$ & 66.7 \\
\hline Data given as mean & \\
\hline & \\
\hline
\end{tabular}

Data given as mean $\pm \mathrm{SD}$ or $\%$. ACEI, angiotensin-converting enzyme inhibitor; ARB, angiotensin II receptor blocker; BMI, body mass index; CCB, calcium channel blocker; DBP, diastolic blood pressure; $\mathrm{HFpEF}$, heart failure with preserved ejection fraction; MMSE, Mini Mental State Examination; SBP, systolic blood pressure.

(1) those who were unable to give written informed consent; (2) those who had difficulty walking; (3) those with a history of dementia; and (4) those unable to undergo the Mini Mental State Examination (MMSE). Demographic data were collected, and blood tests were done just before discharge from hospital.

\section{Echocardiography}

Echocardiography was also performed just before discharge from hospital, using a Vivid E9 (GE healthcare, USA) with a 3.5-MHz transducer. All echocardiography was done by experienced sonographers. Conventional echocardiography data were recorded according to the guidelines of the American Society of Echocardiography. ${ }^{10,11} \mathrm{We}$ obtained diastolic interventricular septal diameter, LV diastolic dimension, and diastolic posterior wall diameter. LVEF and left atrial volume (LAV) were measured using the modified Simpson's method. Transmitral inflow was measured on B-mode imaging in the apical 3-chamber view.

\section{Body Composition}

Muscle weight and body water volume was evaluated using a bioelectrical impedance analyzer (Inbody S10, Inbody Japan, Tokyo, Japan) just before discharge from hospital. The electric leads of the device were attached to the 4 limbs of the patient in the supine position, and demographic data

\begin{tabular}{|lc|}
\hline Table 2. Serum Parameters & \\
Hemoglobin (mg/dL) & $12.2 \pm 2.1$ \\
Hematocrit (\%) & $37.5 \pm 6.2$ \\
Total protein (mg/dL) & $6.9 \pm 0.9$ \\
Albumin (mg/dL) & $3.1 \pm 0.5$ \\
Total bilirubin (mg/dL) & $0.7 \pm 0.3$ \\
BUN (mg/dL) & $25.1 \pm 12.2$ \\
Creatinine (mg/dL) & $1.1 \pm 0.6$ \\
eGFR (mL/min/1.73 $\left.\mathrm{m}^{2}\right)$ & $47.4 \pm 15.2$ \\
Na (mEq/L) & $140.1 \pm 3.6$ \\
$\mathrm{~K}(\mathrm{mEq} / \mathrm{L})$ & $4.1 \pm 0.4$ \\
Cl (mEq/L) & $103.2 \pm 4.7$ \\
Uric acid (mg/dL) & $6.8 \pm 1.9$ \\
BNP (pg/mL) & $372.4 \pm 308.0$ \\
Blood glucose (mg/dL) & $107 \pm 31$ \\
Serum osmolality (mOsm) & $293 \pm 7$ \\
\hline
\end{tabular}

Data given as mean \pm SD. BNP, brain natriuretic peptide; BUN, blood urea nitrogen; eGFR, estimated glomerular filtration rate.

(age, height, body weight, and sex) were entered into the apparatus. Then the device automatically assessed the muscle weight, total body water (TBW) volume, ECW, intracellular body water, and body fat ratio. Validation of the Inbody S10 has been reported previously.12,13 In order to assess the severity of edema, the ratio of ECW to TBW $(\mathrm{ECW} / \mathrm{TBW})$ was calculated.

\section{MMSE and Laboratory Tests}

To evaluate the cognitive function of the patients admitted with HF, MMSE evaluation was carried out by the third author (C.J.) after stabilization before discharge from hospital. Serum brain natriuretic peptide (BNP) was measured using an enzyme immunoassay (E test TOSOH II (BNP); TOSOH, Tokyo, Japan) with a coefficient of variation $<15 \%$.

\section{Informed Consent}

The hospital institutional review board approved this study (approval no., C-T2019-0004). We obtained written informed consent from the patients. When cognitive function was moderately impaired, written informed consent was obtained from the family.

\section{Statistical Analysis}

Results are given as mean \pm SD for continuous variables or as percentage for categorical variables. Correlations between MMSE score and demographic factors, laboratory data, body composition variables, and echocardiography parameters were evaluated using Pearson's (continuous variables) or Spearman's (categorical variables) correlation coefficients. Independent associations between MMSE score and demographic factors or body composition variables were assessed using stepwise multiple linear regression analysis using the following 2 models: model 1 adjusted for demographic factors and body composition variables, and model 2 adjusted for echocardiography parameters in addition to model 1 . The results of stepwise multiple linear regression analysis are given as unstandardized coefficients (B) and 95\% CI and as standardized $\beta$ coefficients. There was no significant collinearity (variance inflation factor was always $<2.0$ ) and no significant interactions were 


\begin{tabular}{|lc|}
\hline \multicolumn{2}{l}{ Table 3. Body Muscle and Water Composition on BIA } \\
ICW $(\mathrm{kg})$ & $14.6 \pm 3.1$ \\
ECW $(\mathrm{kg})$ & $10.3 \pm 1.9$ \\
TBW $(\mathrm{kg})$ & $24.82 \pm 4.99$ \\
Total muscle $(\mathrm{kg})$ & $30.9 \pm 7.0$ \\
BMI $\left(\mathrm{kg} / \mathrm{m}^{2}\right)$ & $20.0 \pm 3.3$ \\
Total fat ratio $(\%)$ & $26.2 \pm 9.2$ \\
ECW/TBW ratio & \\
Whole body & $0.414 \pm 0.014$ \\
ECW/TBW ratio & \\
Right upper limb & $0.388 \pm 0.008$ \\
Left upper limb & $0.389 \pm 0.008$ \\
Trunk & $0.416 \pm 0.015$ \\
Right lower limb & $0.419 \pm 0.016$ \\
Left lower limb & $0.419 \pm 0.015$ \\
\hline
\end{tabular}

Data given as mean \pm SD. BIA, bioelectrical impedance analysis; BMI, body mass index; ECW, extracellular body water; ICW, intracellular body water; TBW, total body water.

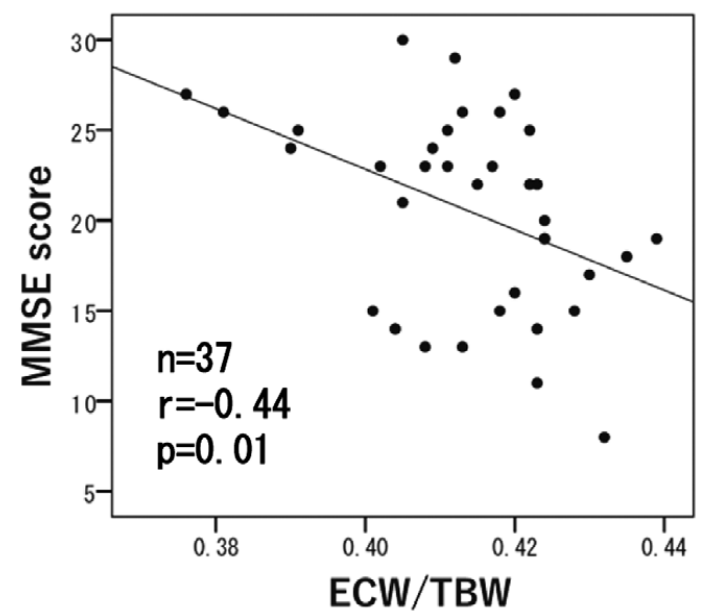

Figure 1. Mini Mental State Examination (MMSE) score vs. extracellular body water/total body water (ECW/TBW) ratio.

\begin{tabular}{|lc|}
\hline \multicolumn{2}{|l|}{ Table 4. Echocardiography Parameters } \\
LVEDD $(\mathrm{mm})$ & $46.5 \pm 8.0$ \\
LVESD $(\mathrm{mm})$ & $35.5 \pm 10.1$ \\
IVS thickness $(\mathrm{mm})$ & $10.7 \pm 2.4$ \\
Posterior wall thickness $(\mathrm{mm})$ & $13.2 \pm 14.8$ \\
EF (modified Simpson's method) $(\%)$ & $46.9 \pm 16.2$ \\
End-diastolic volume $(\mathrm{mL})$ & $87.7 \pm 43.1$ \\
End-systolic volume $(\mathrm{mL})$ & $51.2 \pm 37.7$ \\
LAD (mm) & $43.6 \pm 7.5$ \\
LAVI (mL/m²) & $60.1 \pm 23.1$ \\
TMF deceleration time $(\mathrm{ms})$ & $191.5 \pm 69.9$ \\
TMF E wave velocity $(\mathrm{cm} / \mathrm{s})$ & $78.8 \pm 28.0$ \\
TMF A wave velocity $(\mathrm{cm} / \mathrm{s})$ & $78.9 \pm 24.9$ \\
Septal e' (cm/s) & $4.4 \pm 1.5$ \\
Lateral e' (cm/s) & $6.0 \pm 2.9$ \\
LVOT velocity-time integral $(\mathrm{cm})$ & $16.0 \pm 11.0$ \\
TRPG (mmHg) & $30.2 \pm 8.5$ \\
IVC diameter (mm) & \\
Inspiratory phase & $7.4 \pm 6.0$ \\
Expiratory phase & $13.1 \pm 5.4$ \\
\hline
\end{tabular}

Data given as mean \pm SD. e', mitral e' velocity; EF, ejection fraction; IVC, inferior vena cava; IVS, interventricular septum; LAD, left atrial diameter; LAVI, left atrial volume index; LVEDD, left ventricular end-diastolic diameter; LVESD, left ventricular end-systolic diameter; LVOT, left ventricular outflow tract; TMF, transmitral flow; TRPG, tricuspid regurgitation pressure gradient.

detected. IBM SPSS (version 25.0; Chicago, IL, USA) was used for all analyses and $\mathrm{P}<0.05$ was considered to indicate statistical significance.

\section{Results}

\section{Subjects}

The demographic characteristics and serum parameters of the 43 subjects are given in Tables $\mathbf{1 , 2}$ respectively. Mean age was $85.1 \pm 8.9$ years and $42.0 \%$ were men. A total of $51.2 \%$ of the enrolled patients had HFpEF. Mean MMSE score was $20.5 \pm 5.4$, with the score being $\leq 23$ in $66.7 \%$ of patients. The proportion of patients with MMSE $\leq 23$ in the HFrEF and the HFpEF groups was $61.9 \%$ and $75.0 \%$, respectively. In addition, $90.7 \%$ of the patients were taking antihypertensive drugs, $18.6 \%$ had diabetes, and $32.6 \%$ had hyperlipidemia. Serum BNP was $372.4 \pm 308.0 \mathrm{pg} / \mathrm{mL}$. Body composition bioelectric impedance measurements are listed in Table $\mathbf{3}$ and echocardiography parameters are listed in Table 4.

\section{MMSE Score Correlations}

MMSE score decreased significantly with the increase in ECW/TBW ratio (Figure 1), which is an indicator of the severity of edema. MMSE score also decreased significantly with increase in age (Figure 2A), transmitral inflow A wave velocity (Figure 2C), and LAV index (LAVI; Figure 2D), but was not correlated with EF. Furthermore, MMSE score was significantly related to regular alcohol drinking (Spearman's $\mathrm{r}=0.44, \mathrm{P}=0.01$ ) and to $\mathrm{LV}$ end-diastolic volume $(\mathrm{r}=0.33, \mathrm{P}=0.0489)$.

On stepwise multiple linear regression analysis, MMSE score was significantly associated with ECW/TBW ratio, even in the model that included age, sex, body mass index, alcohol drinking, current smoking, dyslipidemia, diabetes, and hypertension and uric acid as explanatory variables (Table 5). In the model that contained echocardiography parameters, the indicators of diastolic dysfunction (transmitral inflow A wave velocity and LAVI) were also significant determinants of lower MMSE score.

\section{Discussion}

In the present study, the prevalence of cognitive impairment in elderly patients with HF was $66.7 \%$ when cognitive impairment was defined as MSSE score $\leq 23$. ECW/TBW ratio, which was measured by BIA, and factors related to LV diastolic function were related to MMSE score.

Cognitive impairment is sometimes observed in elderly patients with HF; the prevalence of cognitive impairment in HF varies widely from $25 \%$ to approximately $70-80 \% .14 .17$ According to the Japanese data reported by Saito et al, $37 \%$ of the elderly patients admitted to hospital due to HF 

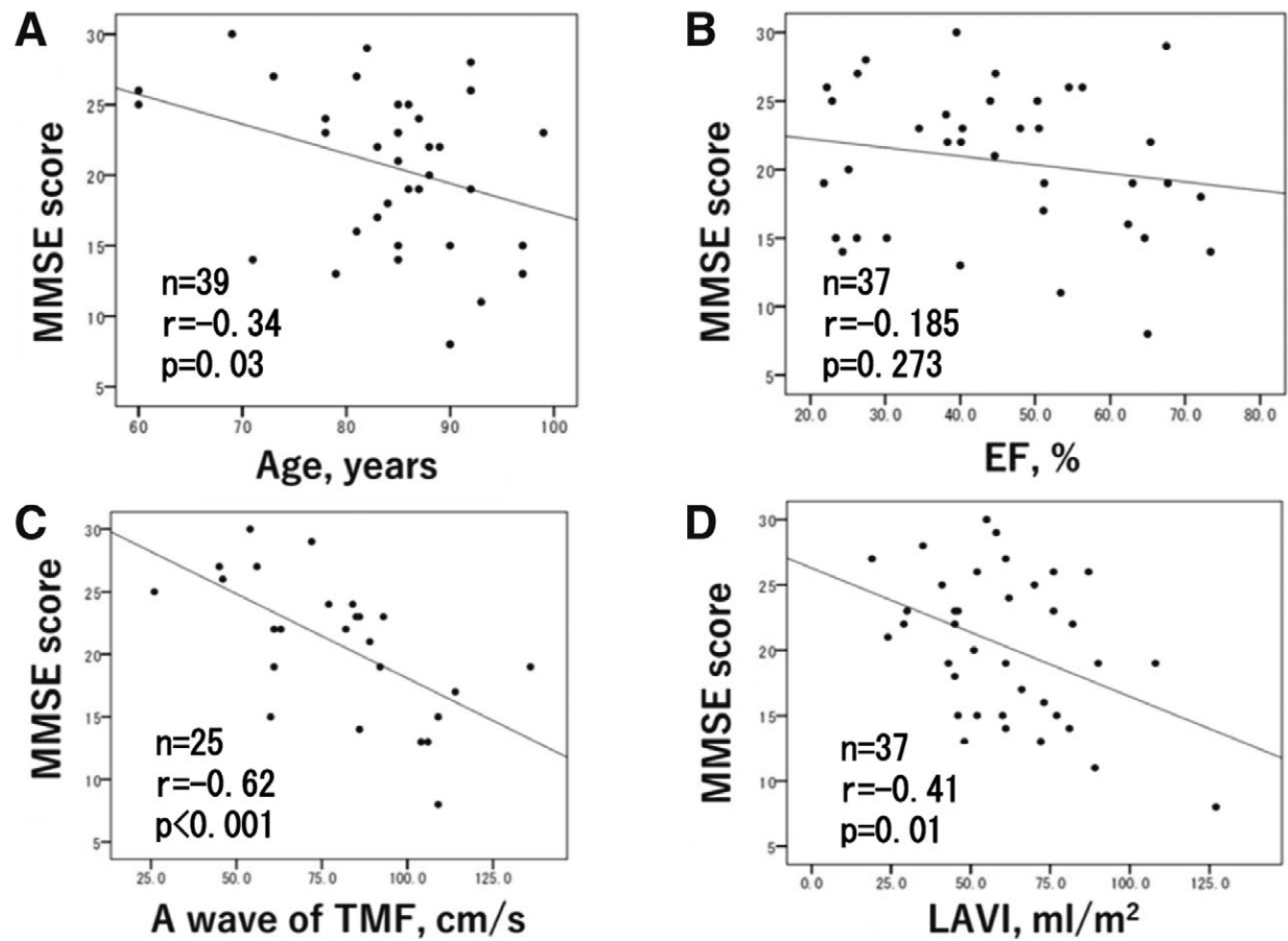

Figure 2. Mini Mental State Examination (MMSE) score vs. (A) age, (B) left ventricular ejection fraction (EF), (C) transmitral flow (TMF) A wave velocity and (D) left atrial volume index (LAVI).

\begin{tabular}{|c|c|c|c|c|}
\hline & $\boldsymbol{\beta}$ & B & $95 \% \mathrm{Cl}$ & P-value \\
\hline \multicolumn{5}{|c|}{ Model 1 (including demographic factors and body composition variables) } \\
\hline ECW/TBW ratio & -0.44 & -180.17 & -311.49 to -48.85 & 0.009 \\
\hline \multicolumn{5}{|c|}{ Model 2 (including echocardiography parameters in addition to model 1) } \\
\hline TMF A wave velocity $(\mathrm{cm} / \mathrm{s})$ & -0.59 & -0.13 & -0.20 to -0.06 & 0.001 \\
\hline $\mathrm{LAVI}\left(\mathrm{mL} / \mathrm{m}^{2}\right)$ & -0.42 & -0.10 & -0.17 to -0.02 & 0.015 \\
\hline
\end{tabular}

Explanatory variables in model 1 were age, sex, body mass index, alcohol drinking, current smoking, dyslipidemia, diabetes, hypertension, atrial fibrillation and uric acid. ECW/TBW, extracellular body water/total body water; LAVI, left atrial volume index; MMSE, Mini Mental State Examination; TMF, transmitral flow.

had cognitive impairment as diagnosed using MMSE score. ${ }^{18}$ The prevalence of cognitive impairment in patients with $\mathrm{HF}$ is affected by the characteristics of the sample, comorbidities and methods used to assess cognition. In the present institute, many patients with HF already had a diagnosis of dementia at the time of admission, and it was difficult to obtain informed consent from those patients. Therefore, we could obtain informed consent only from those patients who did not have a previous history of cognitive impairment. Although it is difficult to assess the general prevalence of cognitive impairment in elderly patients with $\mathrm{HF}$, the prevalence of cognitive impairment in the present study was similar to these previous studies. ${ }^{14-18}$

Cognitive impairment makes it more difficult to manage the conditions of elderly patients with HF because it leads to difficult behavior, reduced medication adherence, missed appointments, and inability to stay on a diet. Therefore, a tool that allows prediction and early detection of cognitive impairment may be useful for managing elderly HF patients.

We found that abnormal LA function (increased transmitral flow A wave velocity and LAVI) was significantly associated with a low MMSE score. The LA has an important role in the pathophysiology and prognosis of HF, and LA enlargement is correlated with LV diastolic dysfunction, and chronic LA pressure and volume overload. ${ }^{19}$ A relationship between cognitive dysfunction and diastolic dysfunction or LA enlargement has been noted. ${ }^{20}$ Sacre et al reported that mild cognitive impairment was twice as frequent in patients with diastolic dysfunction compared with those with normal cardiac structure/function. ${ }^{6}$ Oh et al identified a positive correlation between LA enlargement with cognitive dysfunction and the extent of cerebral white matter changes. ${ }^{21}$ In addition, Karadag et al reported that LAVI $\geq 34 \mathrm{~mL} / \mathrm{m}^{2}$ was independently associated with 
cognitive dysfunction. ${ }^{22}$ The mechanism of the association between cognitive impairment and atrial dysfunction has not been elucidated, but the two main possibilities are (1) silent cerebral infarction caused by atrial fibrillation (AF);23 and (2) reduced cerebral perfusion due to low cardiac output. ${ }^{24}$ In the present study, 16 patients had AF of any type $(37.2 \%)$, but the number of AF patients was too small for sufficient evaluation of the association between cognitive impairment and AF.

Increased LA pressure causes pulmonary venous congestion, which eventually leads to chronic right HF. A high $\mathrm{ECW} / \mathrm{TBW}$ ratio indicates an increase of edema, which is one of the characteristics of right HF. We found that $\mathrm{ECW} / \mathrm{TBW}$ ratio was correlated with cognitive impairment, which may indicate a relationship between abnormal LA function and cognitive impairment. Elevation of the ECW/ TBW ratio suggests the development of organ edema, therefore it is possible that cerebral edema induced cognitive impairment in the present patients, although we could not estimate the cerebral water distribution in this study. Advances in diffusion MRI have enabled the estimation of extracellular free water. ${ }^{25}$ Therefore, a future investigation is needed to determine the cerebral water content on brain MRI and assess the relationship between cerebral edema, cognitive impairment, and HF.

In the present study, on bioelectrical impedance analysis using the Inbody S10, a lower MMSE score was associated with an increase of TBW relative to the extracellular area. The body fluid status of HF patients is usually estimated by clinical assessment based on body weight, the degree of edema, echocardiography parameters, and data from right heart catheterization. However, some of these methods are subjective and imprecise, while catheterization is invasive. Bioelectrical impedance analysis is an easy-to-use, noninvasive and non-expensive method of estimating body fluid status that can be performed at the bedside. According to the present study, this method might be a helpful tool for predicting the risk of cognitive impairment in elderly patients with HF.

\section{Study Limitations}

This was a pilot study performed at a single center, and the number of subjects was small. Accordingly, we need to confirm the results using a multicenter study in a larger number of subjects. In addition, we did not distinguish AF patients from non-AF patients due to the small number of subjects, although the previous studies have identified a relationship between $\mathrm{AF}$ and cognitive dysfunction. Therefore, evaluation of the relationship between ECW/TBW ratio and cognitive dysfunction in a larger study with both $\mathrm{AF}$ and non-AF patients is necessary. Furthermore, we assessed the body fluid and muscle status using the bioelectrical impedance method. However, the gold standard for measurement of muscle is dual energy X-ray absorptiometry (DEXA), and the bioelectrical impedance device that we used (Inbody S10) has not been sufficiently validated, and comparison with DEXA is also needed. Finally, this was a cross-sectional study and the information we obtained could not be used to assess causal relationships. Therefore, longitudinal data on the changes in cognitive function in such patients need to be obtained in the future.

\section{Conclusions}

Cognitive impairment in elderly HF patients might be influenced by the distribution of body water and abnormal LA function. Evaluation of body fluid status using the bioelectrical impedance method could be helpful for assessing the risk of cognitive impairment in HF patients, but further investigation is needed to confirm the association and the underlying mechanism.

\section{Disclosures}

The authors declare no conflicts of interest.

\section{References}

1. Jefferson AL, Himali JJ, Beiser AS, Au R, Massaro JM, Seshadri $\mathrm{S}$, et al. Cardiac index is associated with brain aging: The Framingham Heart Study. Circulation 2010; 122: 690-697.

2. Jefferson AL, Himali JJ, Au R, Seshadri S, Decarli C, O'Donnell $\mathrm{CJ}$, et al. Relation of left ventricular ejection fraction to cognitive aging (from the Framingham Heart Study). Am J Cardiol 2011; 108: $1346-1351$.

3. Sugie M, Harada K, Takahashi T, Nara M, Kawai H, Fujiwara Y, et al. Peak exercise stroke volume effects on cognitive impairment in community-dwelling people with preserved ejection fraction. ESC Heart Fail 2018; 5: 876-883.

4. Sabayan B, van Buchem MA, Sigurdsson S, Zhang Q, Harris $\mathrm{TB}$, Gudnason V, et al. Cardiac hemodynamics are linked with structural and functional features of brain aging: The age, gene/ environment susceptibility (AGES) Reykjavik Study. $J$ Am Heart Assoc 2015; 4: e001294.

5. van den Hurk K, Reijmer YD, van den Berg E, Alssema M, Nijpels G, Kostense PJ, et al. Heart failure and cognitive function in the general population: The Hoorn Study. Eur J Heart Fail 2011; 13: 1362-1369.

6. Sacre JW, Ball J, Wong C, Chan YK, Stewart S, Kingwell BA, et al. Mild cognitive impairment is associated with subclinical diastolic dysfunction in patients with chronic heart disease. Eur Heart J Cardiovasc Imaging 2018; 19: 285-292.

7. Iida N, Seo Y, Sai S, Machino-Ohtsuka T, Yamamoto M, Ishizu T, et al. Clinical implications of intrarenal hemodynamic evaluation by Doppler ultrasonography in heart failure. JACC Heart Fail 2016; 4: 674-682.

8. Dupont M, Mullens W, Tang WH. Impact of systemic venous congestion in heart failure. Curr Heart Fail Rep 2011; 8: $233-$ 241.

9. Verbrugge FH, Dupont M, Steels P, Grieten L, Malbrain M, Tang WH, et al. Abdominal contributions to cardiorenal dysfunction in congestive heart failure. J Am Coll Cardiol 2013; 62: $485-495$.

10. Sahn DJ, DeMaria A, Kisslo J, Weyman A. Recommendations regarding quantitation in M-mode echocardiography: Results of a survey of echocardiographic measurements. Circulation 1978; 58: $1072-1083$.

11. Schiller NB, Shah PM, Crawford M, DeMaria A, Devereux R, Feigenbaum $\mathrm{H}$, et al. Recommendations for quantitation of the left ventricle by two-dimensional echocardiography: American Society of Echocardiography Committee on Standards, Subcommittee on Quantitation of Two-Dimensional Echocardiograms. J Am Soc Echocardiogr 1989; 2: 358-367.

12. Buckinx F, Reginster JY, Dardenne N, Croisiser JL, Kaux JF, Beaudart $\mathrm{C}$, et al. Concordance between muscle mass assessed by bioelectrical impedance analysis and by dual energy X-ray absorptiometry: A cross-sectional study. BMC Musculoskelet Disord 2015; 16: 60.

13. Martinoli R, Mohamed EI, Maiolo C, Cianci R, Denoth F, Salvadori S, et al. Total body water estimation using bioelectrical impedance: A meta-analysis of the data available in the literature. Acta Diabetol 2003; 40(Suppl 1): s203-s206.

14. Leto L, Feola M. Cognitive impairment in heart failure patients. J Geriatr Cardiol 2014; 11: 316-328.

15. Harkness K, Demers C, Heckman GA, McKelvie RS. Screening for cognitive deficits using the Montreal cognitive assessment tool in outpatients $>/=65$ years of age with heart failure. Am J Cardiol 2011; 107: 1203-1207.

16. Athilingam P, D'Aoust RF, Miller L, Chen L. Cognitive profile in persons with systolic and diastolic heart failure. Congest Heart Fail 2013; 19: 44-50.

17. Vogels RL, Scheltens P, Schroeder-Tanka JM, Weinstein HC. Cognitive impairment in heart failure: A systematic review of the literature. Eur J Heart Fail 2007; 9: 440-449. 
18. Saito H, Matsue Y, Suzuki M, Kamiya K, Hasegawa Y, Endo Y, et al. Discordance between subjective and objective evaluations of cognitive function in old Japanese patients with heart failure. Australas J Ageing 2019; 38: 57-59.

19. Zile MR, Gottdiener JS, Hetzel SJ, McMurray JJ, Komajda M, McKelvie R, et al. Prevalence and significance of alterations in cardiac structure and function in patients with heart failure and a preserved ejection fraction. Circulation 2011; 124: 2491-2501.

20. Arangalage D, Ederhy S, Dufour L, Joffre J, Van der Vynckt C, Lang S, et al. Relationship between cognitive impairment and echocardiographic parameters: A review. J Am Soc Echocardiogr 2015; 28: 264-274.

21. Oh JE, Shin JW, Sohn EH, Jung JO, Jeong SH, Song HJ, et al. Effect of cardiac function on cognition and brain structural changes in dementia. J Clin Neurol 2012; 8: 123-129.
22. Karadag B, Ozyigit T, Ozben B, Kayaoglu S, Altuntas Y. Relationship between left atrial volume index and cognitive decline in elderly patients with sinus rhythm. J Clin Neurosci 2013; 20: $1074-1078$.

23. Ott A, Breteler Monique MB, de Bruyne Martine C, van Harskamp F, Grobbee Diederick E, Hofman A. Atrial fibrillation and dementia in a population-based study. Stroke 1997; 28: 316-321.

24. Patel DA, Lavie CJ, Milani RV, Shah S, Gilliland Y. Clinical implications of left atrial enlargement: A review. Ochsner J 2009; 9: $191-196$.

25. Lesh TA, Maddock RJ, Howell A, Wang H, Tanase C, Daniel Ragland J, et al. Extracellular free water and glutathione in first-episode psychosis: A multimodal investigation of an inflammatory model for psychosis. Mol Psychiatry, doi:10.1038/ s41380-019-0428-y. 